\title{
What's in a name? That which we call IPF, by any other name would act the same
}

\author{
Athol U. Wells ${ }^{1}$, Kevin K. Brown ${ }^{2}$, Kevin R. Flaherty ${ }^{3}$, Martin Kolb ${ }^{4}$ and \\ Victor J. Thannickal ${ }^{5}$, on behalf of the IPF Consensus Working Group ${ }^{6}$ \\ Affiliations: \\ ${ }^{1}$ Interstitial Lung Disease Unit, Royal Brompton Hospital, London, UK. \\ ${ }^{2}$ National Jewish Health, Denver, CO, USA. \\ ${ }^{3}$ University of Michigan Health System, Ann Arbor, MI, USA. \\ ${ }^{4}$ Dept of Respiratory Medicine, Pathology and Molecular Medicine, McMaster University, Hamilton, ON, \\ Canada. \\ ${ }^{5}$ Division of Pulmonary, Allergy and Critical Care Medicine, University of Alabama at Birmingham, \\ Birmingham, AL, USA. \\ ${ }^{6} \mathrm{~A}$ list of members of the IPF Consensus Working Group can be found in the acknowledgements section.
}

Correspondence: Athol U. Wells, Royal Brompton Hospital, Sydney St, Chelsea, London SW3 6HP, UK. E-mail: rbhildarbht.nhs.uk

@ERSpublications

IPF remains a truly idiopathic fibrotic lung disease, despite suggestions that it is non-idiopathic. There is an urgent need to explore the "lumping" of IPF with other forms of progressive lung fibrosis in clinical research and treatment trials

http://ow.ly/DtyS30jPqR5

Cite this article as: Wells AU, Brown KK, Flaherty KR, et al. What's in a name? That which we call IPF, by any other name would act the same. Eur Respir J 2018; 51: 1800692 [https://doi.org/10.1183/ 13993003.00692-2018].

ABSTRACT Idiopathic pulmonary fibrosis (IPF) remains a truly idiopathic fibrotic disease, with a modest genetic predilection and candidate triggers but no overall explanation for the development of disease in non-familial cases. Agreement on terminology has contributed to major clinical and translational advances since the millennium. It is likely that the entity currently captured by the term "IPF" will be radically reclassified over the next decade, either through "splitting" (into IPF subgroups responding selectively to individual disease-modifying agents) or through "lumping" of IPF with other forms of progressive fibrotic lung disease (with shared pathogenetic mechanisms and IPF-like disease behaviour). In this perspective, we summarise the clinical and pathogenetic justification for a focus on "the progressive fibrotic phenotype" in future clinical and translational research. By this means, we can hope to address the needs of non-IPF patients with inexorably progressive fibrotic disease, currently disenfranchised by lack of access to agents that are efficacious in IPF. In this regard, ongoing trials of antifibrotic therapies in non-IPF patients with progressive fibrosis may be highly influential. Future revision of IPF nomenclature may be warranted if there are major conceptual changes but without compelling justification, the benefits of renaming IPF are likely to be outweighed by the resulting confusion. 


\section{Introduction}

Sir Isaac Asimov once observed that "The card-player begins by arranging his hand for maximum sense. Scientists do the same with the facts they gather." This essential truth has governed historical changes in the definition of idiopathic pulmonary fibrosis (IPF). As defined before the millennium, the "IPF" label included a group of diverse idiopathic interstitial pneumonias (IIPs) that are now viewed as separate entities. The justification for a refined IIP reclassification in 2002 [1] lay in accumulated experience that established that other IIPs had, on average, a radically different outcome from the entity now termed IPF [2]. In the words of William James, a founding father of the pragmatic philosophy movement, "every way of classifying a thing is but a way of handling it for some particular purpose." New nomenclature for a disease is justified by clinical utility: idiopathic non-specific interstitial pneumonia (NSIP) was separated from IPF based on major differences in the natural history and treated course. Whilst our conception of IPF has changed greatly in recent decades, with the 2011 diagnostic criteria [3] currently under revision, both defining and classifying IPF is likely to remain a "moving target".

As discussed in detail below, the future definition and taxonomy of IPF may be driven either by "splitters" (who prefer to draw distinctions from observed detail) or "lumpers" (who search for a big picture over and above diagnostic nuance). The tension between splitting and lumping should be viewed as a source of healthy debate. In IPF, the dilemma can be summarised briefly. With our current approaches to treatment, will unique outcomes be recognised in distinct patient subgroups? Or will the recognition of a clinically progressive fibrotic phenotype, with the amalgamation of IPF patients and non-IPF patients with IPF-like behaviour, prove to be more fruitful? To argue for the primacy of either approach assumes a level of knowledge that we do not yet possess. Indeed, splitting and lumping are arguably legitimate complementary strategies. "Smarter lumping and smarter splitting" were recently advocated for trial design in sepsis and acute respiratory distress syndrome [4]. The only certainty is that the future shape of IPF is uncertain.

It is in this context that we view with some scepticism the recent proposal for a change in nomenclature, with the term "IPF" replaced, it is suggested, by "epithelial-driven pulmonary fibrosis", "primary pulmonary fibrosis" or "progressive age-dependent pulmonary fibrosis" [5]. In themselves, these very different proposed alternative terms capture the enigmatic and idiopathic nature of IPF pathogenesis. The authors argue that the word "idiopathic" is now obsolete. They base this assertion on a perception that there is "an extensive understanding of the genetic, environmental, cellular, and molecular mechanisms involved in the development of IPF". They highlight the current favoured IPF pathogenetic hypothesis that fibrosis results primarily from epithelial cell dysfunction. They question also the term "fibrosis" on the grounds that fibrosis is but one component of IPF, with matrix accumulation resulting from dysfunctional epithelia. They argue that the use of the term "fibrosis" neglects the other pathogenetic processes that contribute to lung remodelling. Based upon these considerations, the authors suggest that the IPF label might limit therapeutic advances and inhibit the implementation of precision medicine.

Although the perspective of WolTERs et al. [5] may provoke fruitful debate, we believe that a change in nomenclature at this moment is premature and likely to have adverse consequences. The designation of a disease as idiopathic does not imply the absence of an underlying cause (or causes) but indicates merely that key triggers of disease are currently unknown or incompletely understood. Much has been learned of the underlying mechanisms in recent years but, as argued elsewhere, the development of IPF in individual patients (except those with familial forms) remains unexplained [6]. In this regard, the identification of a genetic predilection for IPF, consisting of a single nucleotide polymorphism (rs35705950) present in 38\% of IPF patients but $9 \%$ of the general population [7], has a pathogenetic explanatory power of only $5 \%$. Indeed, if it is argued that all chronic disease processes result from a combination of genetic predilection and environmental factors, the disclosure of a modest IPF association with muc5B positivity does not, in itself, unravel the enigma that is IPF.

The view that epithelial cell dysfunction is a key IPF pathogenetic pathway was first given wide currency by a pivotal review article in 2001 [8]. Wolters et al. [5] argue that IPF can now be viewed as essentially an epithelial disease. However, pathobiological mechanisms recognised in IPF include epithelial cell dysfunction, impaired host defence, T-cell exhaustion, fibroblast activation, oxidative stress, vascular remodelling, alternative macrophage activation and senescence/ageing [9] and the interplay between these pathways is likely to be pivotal. For example, the relationship between very rare mutations in genes associated with telomere homeostasis (such as TERT, TERC, PARN or RTEL1) or surfactant homeostasis (such as SFTPC, SFTPA1 and SFTPA2) and the development of pulmonary fibrosis is quite strong and supports a pathogenetic link between senescence and alveolar epithelial abnormalities in pulmonary fibrosis development $[10,11]$. Moreover, the mechanisms responsible for disease initiation may differ from that of progression. For example, cancer progression is critically dependent on the tumour microenvironment, including the stromal and immune cells, despite the epithelial cell origin of most 
cancers. IPF progression may be driven by aberrant cell phenotypes, including epigenetically reprogrammed mesenchymal cells [12], that are distinct from an initiating event such as epithelial cell injury or impaired regeneration. Indeed, there is support for both immune mechanisms [13, 14] and stromal mechanisms [15-18] contributing to fibrosis progression. Furthermore, epithelial abnormalities are likely to be pathogenetically important in most other fibrotic lung diseases, including hypersensitivity pneumonitis, connective tissue disease-associated interstitial lung disease (CTD-ILD) and sarcoidosis, undermining the argument for "epithelial-driven pulmonary fibrosis" as an alternative term to IPF.

We also question the view that the term "fibrosis" is an inappropriate descriptor in IPF. It is fibrosis that is lethal in IPF. Fibrotic disease in IPF is on average more extensive and more progressive than in other interstitial lung diseases, such as fibrotic hypersensitivity pneumonitis and idiopathic fibrotic non-specific interstitial pneumonia, disorders in which fibrogenesis results, at least in part, from immune dysregulation. Pathogenetic arguments do not, we suggest, trump the clinical importance of fibrosis in IPF.

The idea that the IPF label is a bar to further advances in our understanding seems counterintuitive. The term "atherosclerosis" has not hindered advances in the understanding of its complex multicellular pathogenesis that extends well beyond the "sclerosis" component. Similarly, the name "cystic fibrosis" has not hampered research initiatives, despite the absence of a major pathogenetic role for either fibrosis or cyst formation. It can be argued that the designation of IPF as "not yet explained" or "self-perpetuated" (i.e. idiopathic), rather than adequately explained by recent advances (i.e. non-idiopathic), is, in itself, a spur towards essential further research. We subscribe to the view that the use of the term "idiopathic" appropriately influences, in the words of WOLTERs et al. [5], "a patient's (or caregiver's) understanding of their disease and expectations for its behaviour and management."

A radical change in disease nomenclature, even when desirable, is a major undertaking that must inevitably lead to short-term confusion. It was generally acknowledged that the change from "bronchiolitis obliterans organising pneumonia" (BOOP) to "cryptogenic organising pneumonia" (COP) [1] was necessary because of the confusion created by the term "BOOP", containing "bronchiolitis obliterans" but designating a radically different disorder from obliterative bronchiolitis. Yet in the next decade, "BOOP" was almost as prevalent a term as "COP" in the medical literature (figure 1).

The IIPs are not all idiopathic, not always interstitial and seldom pneumonias, but the term has been retained because there is a widespread understanding of, and clinical utility to, its meaning with the vast majority of publications since 2002 based on the 2002 IIP criteria [1]. The intrinsic inaccuracy of the term "IIP" was not a bar to the refinement and greater understanding of these diseases. The prevailing view in the 2013 update of the IIP classification [19] was that a gratuitous change in nomenclature would achieve little of practical value.

Thus it is with IPF. As shown in table 1, a large number of terms have been used historically to describe idiopathic forms of pulmonary fibrosis, in itself a major source of confusion. The unification of terminology in 2002 [1] helped to pave the way for major subsequent advances. There is now universal international recognition of the clinical nature and behaviour of the disease captured by the term "IPF". If it transpires that a subgroup of IPF patients have a major improvement in outcome with a specific agent, selected on the basis of biomarker signal, a taxonomic change in that patient subgroup may indeed be warranted, exactly as happened with the emergence of NSIP as a separable disorder. In that case, the current proposed change from IPF to an alternative term will be followed by the need for a further name change. That, we believe, is a certain pathway to chaos.

FIGURE 1 The frequency of the terms "bronchiolitis obliterans organising pneumonia" (BOOP) and "cryptogenic organising pneumonia" (COP) before and after the publication of the 2002 classification of the idiopathic interstitial pneumonias.

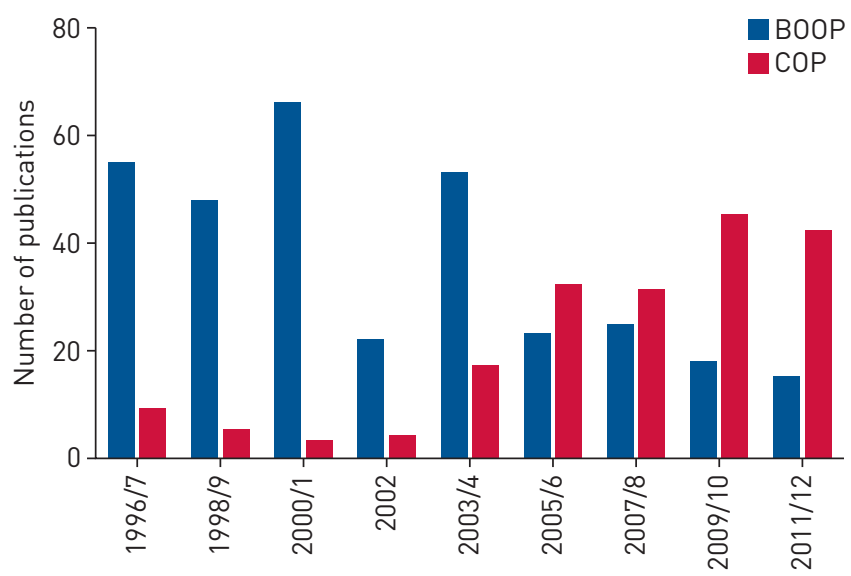


TABLE 1 Historical terms used for "IPF" before consensus was achieved on terminology after the millennium

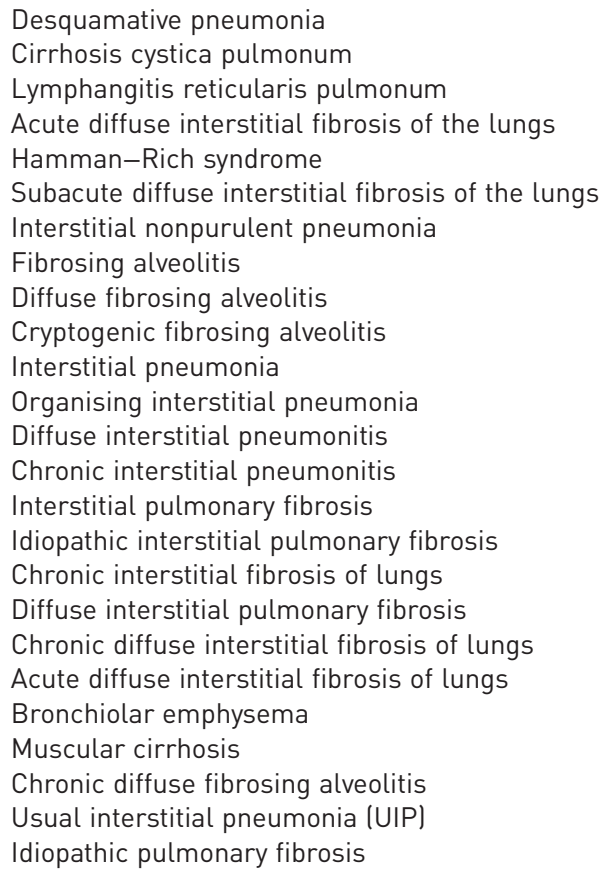

Above all, recent advances, including the many achieved by co-authors of the WolTERs et al. [5] perspective, have not carried us to a final understanding of IPF pathogenesis. The IPF jigsaw has not been solved and may or may not be solved in the near future. Our fundamental objection to the proposed name change is that it is the wrong initiative at a time when priority should instead be given to the possible splitting of IPF in order to implement precision medicine and, in parallel, the lumping of IPF with other forms of progressive fibrosis.

\section{Splitting IPF and precision medicine}

Precision medicine is predicated, in large part, on the discovery of reliable and reproducible biomarkers. In this specific setting, biomarkers can be broadly defined as objectively measured indicators of the underlying disease mechanisms that are informed by the combination of -omics approaches (e.g. genomics, transcriptomics, metabolomics, proteomics and the microbiome) [20, 21], specific proteins and/ or their modifications in circulating plasma [22-26], and lung tissue-based sampling/analysis [26]. The hope that biomarkers, linked to key mechanistic pathways, might identify IPF subgroups selectively responsive to targeted therapies remains unrealised. Currently the only IPF data that provide "proof of concept" come from a retrospective study in a small cohort, in which good and poor outcomes with antioxidant therapy were linked to a TOLLIP gene variant [20]. This is not to deny the importance of precision medicine as a goal in IPF treatment, an idea developed in several influential perspectives [27-30]. However, a note of caution must be sounded. Precision medicine is conceptually elegant but the history of science is littered with elegant ideas, such as the Greek theory of the four humours, that ultimately proved to be unsustainable.

The precedent of efficacious targeted treatment in malignant disease largely relates to interventions directed towards tumour promoter pathways that are aberrantly activated by specific gene mutations. Successful personalised therapies in non-malignant pulmonary diseases, perhaps more relevant to IPF, include ivacaftor in individuals with cystic fibrosis carrying the G551D mutation(s) [31], and mepolizumab and benralizumab in eosinophilic asthma [32, 33]. There are conflicting data on the potential role of lebrikizumab in an asthma subgroup defined by type 2 helper T-cell (Th2)-driven inflammation $[34,35]$. These observations can only be seen as encouraging. However, whether a similar approach will be equally effective in IPF in the near term remains uncertain.

Fibrosis is a complex, yet highly conserved, tissue repair response to injury that becomes maladaptive or manifests as a failure of tissue regenerative capacity [9]. It is viewed as the dominant disease pattern when 
it predominates histologically, as in IPF. However, fibrosis can complicate most ILDs, as well as asthma and chronic obstructive pulmonary disease, and may identify general disease endotypes. The challenge is to link an endotype with longitudinal clinical behaviour. The lack of identification of endotype-phenotype subgroups may explain the failure of many clinical trials in IPF. Until such endotype-phenotype subgoups are identified and verified in IPF, the subclassification ("splitting") of IPF cannot serve a useful clinical purpose.

\section{Lumping IPF with other fibrotic diseases: the progressive fibrotic phenotype}

The intense efforts directed towards the splitting of IPF have not been mirrored by initiatives to identify mechanisms common to IPF and other forms of progressive fibrotic ILD. Specifically, there may be similarities between IPF and other progressive fibrotic ILDs that relate to convergence of initiating precipitants on downstream biological pathways, and grouping, or lumping, these disorders based on these final common pathways may have clinical utility. Over time, IPF has been defined more and more narrowly, carefully separating it from other forms of ILD, and comparing it with control groups separated into individual disorders [36] or amalgamated [37]. This strict phenotyping has not been without drawbacks. For example, screen failure rates in some interventional IPF trials have exceeded 50\%, with the majority of excluded patients failing to meet strict diagnostic criteria [38]. The rate of progression associated with the narrowly defined chest imaging patterns required for a definite diagnosis of IPF does not differ from that associated with more broadly defined patterns [39]. The entity of unclassified IIP, a source of major management uncertainty, was necessary to provide a diagnostic home for patients who failed to meet rigorous classification criteria for a diagnosis of IPF or another IIP [19].

Disease progression in IPF is more common than in other ILDs, albeit at a highly variable rate, and this clinical behaviour is a defining feature of the disease. As IPF is the most common of the fibrosing ILDs, IPF studies at referral centres are comparatively easy to power and have reasonably clear diagnostic criteria. By contrast, the evaluation of disease progression in other individual ILDs complicated by fibrosis requires the selection of underpowered patient subgroups, necessitating multicentre collaboration and requiring robust definitions of disease and progression. It was, perhaps, inevitable that research into mechanisms of progressive pulmonary fibrosis would focus on IPF.

The IPF model of disease progression has the benefit of diagnostic clarity but fails to meet current clinical needs in non-IPF fibrosing lung disease patients. The primary utility of a secure diagnosis of an individual ILD is that it informs clinicians and patients of the expected untreated natural history as well as the treated course of disease. To diagnose IPF is to diagnose an inexorably progressive fibrotic disorder. However, in other ILDs, there is considerably more variability in the course of disease. In hypersensitivity pneumonitis, for example, there are patient subgroups in which disease is fully reversible (whether self-limited or requiring treatment), fibrotic but inherently stable ("burnt-out" disease), fibrotic and progressive but able to be stabilised by traditional therapy, and inexorably progressive. These patterns of longitudinal disease behaviour also apply to connective tissue disease-associated ILD, sarcoidosis, and other forms of ILD (including unclassifiable disease) and are captured in the ILD disease behaviour classification, first proposed in 2003 [40] and formally recommended in a modified form by an American Thoracic Society/ European Respiratory Society expert group for use in unclassifiable ILD [19]. In this perspective, we focus on patient subgroups in non-IPF fibrosing disorders that exhibit disease progression despite treatment. The amalgamation of this patient subset across individual ILDs, hereafter termed "the progressive fibrotic phenotype", is amply justified by clinical data and supported by translational observations (table 2).

\section{Clinical data}

Longitudinal behaviour in fibrotic ILD can usefully be subdivided into three of the five patterns specified in the disease behaviour classification [19].

Pulmonary fibrosis may be intrinsically non-progressive (category 3 of the IIP disease behaviour classification), as in "burnt out" sarcoidosis or residual damage after removal of a trigger (e.g. drug-induced lung disease, some cases of hypersensitivity pneumonitis).

In another large patient subgroup, progressive disease is stabilised by immunomodulation, at least in the short-term (category 4 of the disease behaviour classification). The "average" outcome observed in a placebo-controlled trial of oral cyclophosphamide [41] and in a comparison of oral cyclophosphamide and mycophenolate mofetil in systemic sclerosis [42] was physiological stability. There are also uncontrolled data documenting stabilisation of disease with mycophenolate mofetil therapy in connective tissue disease-associated ILD [43] and chronic hypersensitivity pneumonitis [44, 45].

The progressive fibrotic phenotype in non-IPF disorders is characterised by progression regardless of treatment considered appropriate in individual ILDs (category 5 of the disease behaviour classification), 
TABLE 2 Data indicative of likely shared pathogenetic mechanisms between idiopathic pulmonary fibrosis (IPF) and other progressive fibrotic lung diseases; and subgroups of patients with diseases other than IPF with a progressive IPF-like fibrotic phenotype

Reference

Data indicative of IPF-like disease progresssion in subgroups of patients with other progressive fibrotic lung diseases

IPF-like outcomes in CHP patients with a histological or CT pattern of UIP

IPF-like outcomes in RA patients with a histological or CT pattern indicative of UIP

IPF-like outcomes in IPAF patients with a histological or CT pattern indiciative of UIP

Outcomes intermediate between IPF and other progressive fibrotic diseases in patients with unclassifiable ILD

Reports of patients with drug-induced lung disease exhibiting a fatal progressive fibrotic phenotype despite drug withdrawal

IPF-like outcomes in patients with idiopathic NSIP with disease progression at 6-12 months (as judged by serial FVC trends)

Linkage between serial decline in FVC and mortality in CHP, SSc-ILD and rheumatoid lung, similar to that seen in IPF

Data indicative of pathogenetic mechanisms common to IPF and other progressive fibrotic lung diseases

Shared genetic predilection for IPF and rheumatoid lung

Similar links between short telomere lengths and mortality in IPF and CHP

Linkage between alveolar epithelial cell dysfunction/injury and pulmonary function decline in IPF and SSc-ILD

Pathobiological mechanisms likely to contribute to disease progression in both IPF and SSc-ILD: alveolar stem cell

exhaustion/cellular senescence, mitochondrial dysfunction, impaired autophagy, epigenetic modifications, and immune

dysregulation

CHP: chronic hypersensitivity pneumonitis; CT: computed tomography; UIP: usual interstitial pueumonia; RA: rheumatoid arthritis; IPAF interstitial pneumonitis with autoimmune features; ILD: interstitial lung disease; NSIP: non-specific interstitial pneumonia; FVC: forced vital capacity; SSc: systemic sclerosis.

including a subgroup with IPF-like disease behaviour and mortality (figure 2) and a subgroup in which fibrosis progresses more insidiously.

Outcomes similar to IPF are often seen in patients with a UIP pattern on biopsy or on chest imaging (high-resolution computed tomography; HRCT). In the two largest chronic hypersensitivity pneumonitis histological series, patients with a histological pattern of usual interstitial pneumonia (UIP) had the worst outcome [46, 47] with a survival similar to that of IPF [46], confirming previous observations [48-50]. Macrocystic honeycombing on HRCT, indicative of a likely UIP pattern, is an independent determinant of mortality in chronic hypersensitivity pneumonitis [51]. In rheumatoid lung, a histological pattern of UIP is a malignant prognostic determinant, with survival similar to IPF in some series [52-54], although not in others $[55,56]$. A pattern of UIP on HRCT was, in one series, associated with survival identical to that in a control IPF cohort [57] and has, in general, been indicative of a poor outcome [58-61]. In the entity of

FIGURE 2 The overlap in longitudinal disease behaviour between idiopathic pulmonary fibrosis (IPF) and other disorders. The size of the circles reflects the approximate prevalence of individual diseases encountered at interstitial lung disease (ILD) centres. IPAF: interstitial pneumonitis with autoimmune features; CTD: connective tissue disease; f-NSIP: fibrotic non-specific interstitial pneumonia; HP: hypersensitivity pneumonitis.

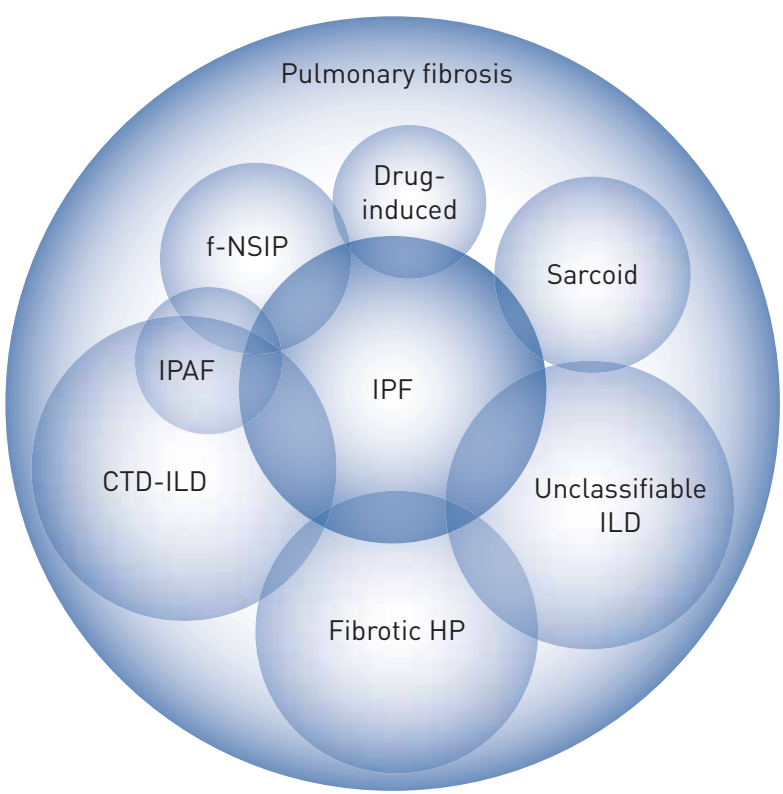


interstitial pneumonitis with autoimmune features (IPAF), defined in a research statement [62], a UIP pattern on biopsy or HRCT has the same outcome as IPF [63].

However, the progressive fibrotic phenotype is not confined to patients with a UIP pattern at surgical biopsy or on HRCT. In rheumatoid lung, a classic UIP HRCT pattern occurs in less than $50 \%$ of patients with a UIP histological pattern [64]. In the remaining cases, HRCT patterns include possible UIP (also compatible with NSIP) and "inconsistent with UIP", as defined in the 2011 American Thoracic Society/ European Respiratory Society/Japanese Respiratory Society/Latin American Thoracic Association guideline [3]. In unclassifiable ILD, survival is intermediate between IPF and other non-IPF ILDs [65-67]. A subgroup of patients with idiopathic NSIP at surgical biopsy, characterised by disease progression over 6 to 12 months, have a mortality rate similar to that of IPF $[68,69]$. There are many anecdotal reports of the evolution of drug-induced lung disease into a fatal progressive fibrotic phenotype, despite withdrawal of the offending agent [70]. Whilst sarcoidosis tends to progress despite treatment more insidiously than other fibrotic ILDs, the 10 -year survival of $84 \%$ associated with stage IV disease on chest radiography is lower than expected in the general population, with mortality mostly ascribable to pulmonary disease [71].

The power of short-term disease behaviour, in identifying adverse long-term outcomes, has been illustrated in studies of NSIP, hypersensitivity pneumonitis, rheumatoid lung, systemic-sclerosis-associated ILD and unclassifiable ILD, in which mortality has been primarily associated with short-term disease progression, defined by pulmonary function trends $[59,68,69,72,73]$ or serial progression of disease on HRCT [65]. In these studies, disease progression was an independent determinant of mortality after other baseline information had been taken into account.

We believe that it would be premature to propose an exact definition of the progressive fibrotic phenotype. Accurate criteria that can be applied at first presentation are highly desirable but would need to be validated prospectively against disease behaviour. Based on current knowledge, summarised in this perspective, it appears likely that a combination of HRCT features indicative of likely UIP, histological findings and emerging molecular data might eventually provide a baseline definition, with additional criteria related to observed disease progression. At present, the progressive fibrotic phenotype can be designated only by observed disease progression, despite treatment considered to be appropriate in individual ILDs.

\section{Pathogenetic considerations}

From a pathophysiological standpoint, fibrosis almost universally results when there is irreparable loss of epithelial or, to a lesser degree, endothelial barrier integrity $[74,75]$, seen in many of the non-IPF ILDs, in particular CTD-ILD and chronic hypersensitivity pneumonitis. Thus, although the aetiology of cellular injury may vary, be it autoimmune mechanisms in CTD-ILD, inhaled organic dust particles in hypersensitivity pneumonitis, or an unknown injury in IPF, the initiation of the fibrotic response is likely to be related to persistent injury and impaired epithelial and/or endothelial cell repair. Similarly, although mechanisms of disease initiation may differ from that of progression, it is highly likely that a conserved spectrum of pathobiological mechanisms, including growth factor activation and epigenetic reprogramming of fibroblasts, underlie the rate of disease progression in various ILDs, despite different underlying aetiologies and initially distinct biological pathways. The same pathobiological mechanisms that contribute to fibrosis progression in IPF may also contribute to a progressive disease phenotype in non-IPF fibrotic disorders such as CTD-ILD and chronic hypersensitivity pneumonitis. Thus, from a pathogenetic perspective, it may be more useful to "lump" these diverse fibrotic disorders and identify endotype-phenotype subgroups to more precisely assign prognosis and to initiate targeted therapies.

Although there is a relative paucity of translational studies exploring linkages between disease progression in IPF and other fibrotic disorders, there are emerging data that common mechanisms may exist. Shared genetic susceptibility in IPF and rheumatoid arthritis-associated ILD has been recently demonstrated, suggesting that common pathogenetic events are involved in these diseases [76]. Short telomere length, which predicts increased mortality in IPF [77], has similar prognostic significance in chronic hypersensitivity pneumonitis [78]. Additionally, serum biomarkers of alveolar epithelial cell injury or dysfunction correlates with subsequent lung function decline in both systemic sclerosis-associated ILD (SSc-ILD) and IPF [79]. Common pathobiological mechanisms that contribute to disease progression in SSc-ILD and IPF might include alveolar stem cell exhaustion/cellular senescence, mitochondrial dysfunction, impaired autophagy, epigenetic modifications and immune dysregulation [80-83].

Disease progression in subgroups of patients with SSc-ILD and IPF may occur independent of the risk of developing the disease, as illustrated by the lack of association of the IPF risk MUC5B gene variant with either SSc-ILD or sarcoidosis [84]. Interestingly, this same single nucleotide polymorphism in MUC5B which confers increased risk of developing IPF is also significantly associated with improved survival [85]. 
Furthermore, a TOLLIP single nucleotide polymorphism is associated with a decreased risk of developing IPF, yet a worse prognosis [86]. Although the reasons for these apparently paradoxical findings are unknown, they further highlight the importance of distinguishing disease risk from disease progression.

\section{The progressive fibrotic phenotype: implications and future strategies}

In this perspective, we have highlighted the potential value of lumping IPF with other forms of progressive fibrosing lung disease, as a counterpoint to the recent suggestion to split IPF, with the ultimate goal of precision, or personalised medicine. The presence of fibrosis is a defining characteristic of a group of progressive lung diseases that includes, but is not limited to IPF. That fibrosis progresses in many patients, and that this progression is responsible for impairment in quality of life, declining functional status and early mortality, is unchallenged. It is likely that a wide variety of mechanisms result in the initiation of disease, while those responsible for its progression remain uncertain. We argue that translational and interventional research, identifying mechanisms responsible for the development and progression of organ fibrosis common to IPF and other fibrotic ILDs offers a more compelling path forward. After 20 years of experience with large multicentred IPF trials, we aver that a rigid approach, requiring that the efficacy of newly identified disease-modifying therapies must be demonstrated by controlled data in each individual ILD, is unrealistic, inefficient, and may disenfranchise non-IPF patients with a similar clinical course to IPF. Similar to the basket trial design in oncology, grouping non-IPF patients with the progressive fibrotic phenotype in pharmaceutical studies, as in ongoing trials of nintedanib and pirfenidone in patients with progressive fibrosis (amalgamating non-IPF disorders) [87, 88], provides a more scientifically rational approach.

Splitting and lumping are best viewed as complementary approaches. Diagnostic biomarkers, believed to be specific to IPF or its subpopulations, should ideally be examined against disease progression in other fibrotic ILDs. Similarly, biomarkers shown to have prognostic value in IPF, thereby defining IPF subgroups, should also be explored in non-IPF patients with fibrosing lung disease. In this regard, lumping and splitting may best be viewed as the yin and yang of pathogenetic thinking in fibrotic ILD.

\section{Conclusion}

In summary, we believe that the term "IPF" should be retained, but recognise that this is an evolving area. The current understanding of IPF will certainly increase with the generation of new knowledge about distinct disease subgroups and key pathobiological pathways that may also be present in other fibrotic ILDs. The current arguments for a change in name are fundamentally unconvincing and there are major concerns about the adverse consequences of a name change at this time. Agreement on terminology,

TABLE 3 Historical milestones in the understanding and management of idiopathic pulmonary fibrosis (IPF)

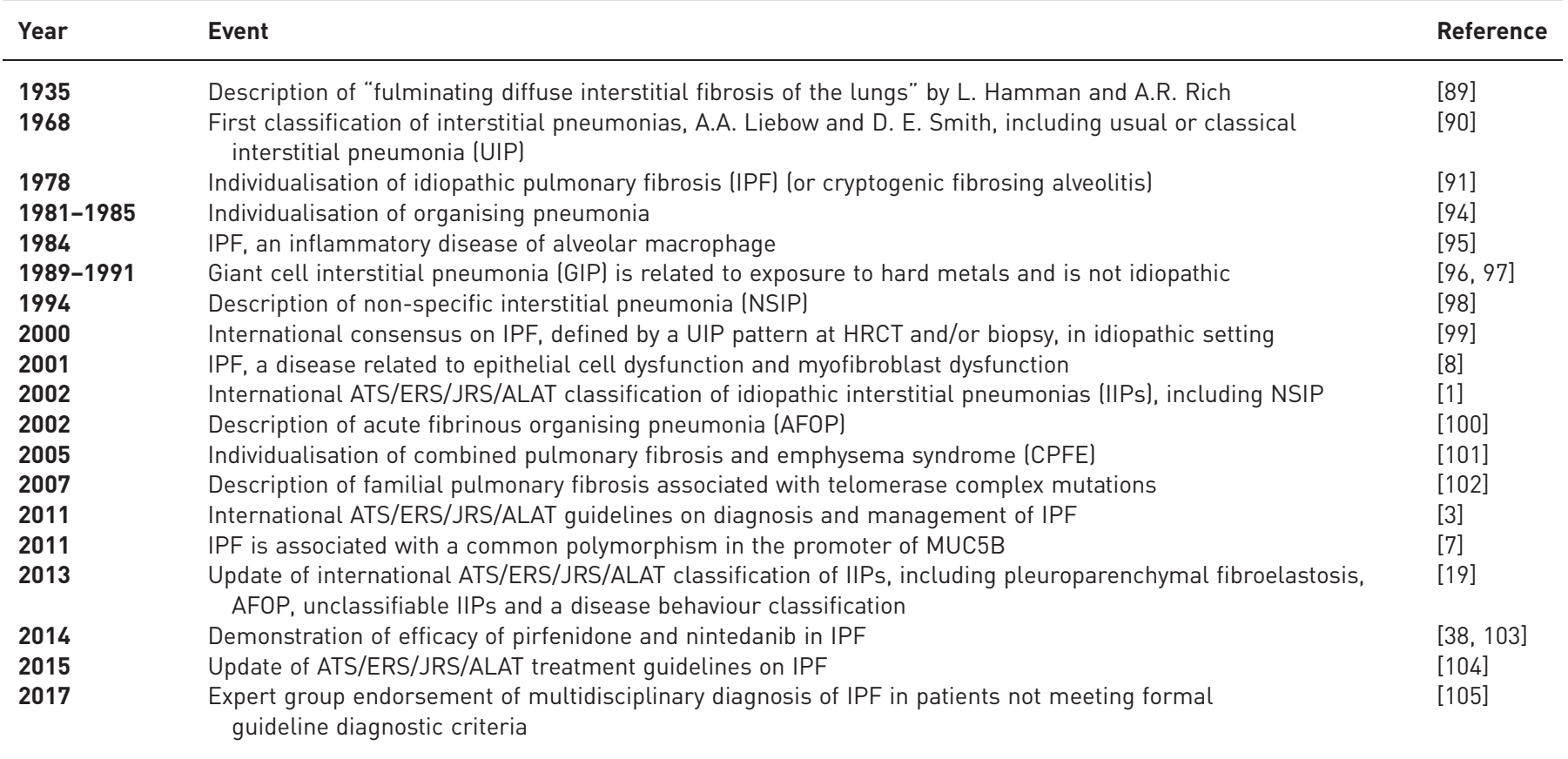


forged after the millennium, is among the major advances in the understanding and management of IPF, summarised in table $3[1,3,7,8,19,38,89-105]$. We suggest that the more important initiative is to expand our definitions and explore the progressive fibrotic phenotype, whilst preserving IPF as a comparator with other, progressive, forms of fibrotic ILD.

The IPF Consensus Working Group: K.M. Antoniou, J. Behr, P.B. Bitterman, C.D. Cool, U. Costabel, T.V. Colby, V. Cottin, B. Crestani, G.P. Downey, J. Gauldie, S.D. Groshong, A. Guenther, J.C. Horowitz, Y. Inoue, J.A. Lasky, A.H. Limper, D.A. Lynch, F.J. Martinez, J.L. Myers, A.G. Nicholson, I. Noth, V. Poletti, C.J. Ryerson, J.H. Ryu, D. Valeyre, C. Vancheri, E.S. White.

Conflict of interest: A.U. Wells reports personal fees for consultancy and lecturing from Intermune/Roche, Boehringer Ingelheim and Bayer, and personal fees for consultancy from Gilead, outside the submitted work. K.K. Brown reports multiple lung fibrosis grants from NHLBI, personal fees from AstraZeneca, Biogen, Fibrogen, Galecto, Gilead, MedImmune, Novartis, Aeolus, ProMetic, Patara, Third Pole, aTyr, Galapagos and Boehringer Ingelheim, has a submitted grant with Roche/Genentech, and conversation under CDA only with Global Blood Therapeutics and Genoa, outside the submitted work. K.R. Flaherty reports grants and personal fees from Boehringer Ingelheim and Roche/ Genentech, grants from Afferent, personal fees from Veracyte, Fibrogen, Immuneworks, Aeolus, Pharmakea, Sanofi-genzyme and Celgene, outside the submitted work. M. Kolb reports grants and personal fees from Roche, Boehringer Ingelheim, GSK and Prometic, personal fees from Gilead and Genoa, and grants from Actelion, Respivert, Alkermes and Pharmaxis, outside the submitted work.

\section{References}

1 American Thoracic Society, European Respiratory Society. American Thoracic Society/European Respiratory Society international multidisciplinary consensus classification of the idiopathic interstitial pneumonias. Am J Respir Crit Care Med 2002; 165: 277-304.

2 Bjoraker JA, Ryu JH, Edwin MK, et al. Prognostic significance of histopathologic subsets in idiopathic pulmonary fibrosis. Am J Respir Crit Care Med 1998; 157: 199-203.

3 Raghu G, Collard HR, Egan JJ, et al. An official ATS/ERS/JRS/ALAT statement: idiopathic pulmonary fibrosis: evidence-based guidelines for diagnosis and management. Am J Respir Crit Care Med 2011; 183: 788-824

4 Prescott HC, Calfee CS, Thompson BT, et al. Toward smarter lumping and smarter splitting: rethinking strategies for sepsis and acute respiratory distress syndrome clinical trial design. Am J Respir Crit Care Med 2016; 194: 147-155

5 Wolters PJ, Blackwell TS, Eickelberg O, et al. Time for a change: is idiopathic pulmonary fibrosis still idiopathic and only fibrotic? Lancet Respir Med 2018; 6: 154-160.

6 Thannickal VJ, Wells A, Kolb M. Idiopathic pulmonary fibrosis: idiopathic no more? Lancet Respir Med 2018; 6: 84-85.

7 Seibold MA, Wise AL, Speer MC, et al. A common MUC5B promoter polymorphism and pulmonary fibrosis. N Engl J Med 2011; 364: 1503-1512.

8 Selman M, King TE, Pardo A, et al. Idiopathic pulmonary fibrosis: prevailing and evolving hypotheses about its pathogenesis and implications for therapy. Ann Intern Med 2001; 134: 136-151.

9 Thannickal VJ, Zhou Y, Gaggar A, et al. Fibrosis: ultimate and proximate causes. J Clin Invest 2014; 124: 4673-4677.

10 Borie R, Kannengiesser C, Sicre de Fontbrune F, et al. Management of suspected monogenic lung fibrosis in a specialised centre. Eur Respir Rev 2017; 26: 160122.

11 Selman M, López-Otín C, Pardo A. Age-driven developmental drift in the pathogenesis of idiopathic pulmonary fibrosis. Eur Respir J 2016; 48: 538-552.

12 Korfei M, Skwarna S, Henneke I, et al. Aberrant expression and activity of histone deacetylases in sporadic idiopathic pulmonary fibrosis. Thorax 2015; 70: 1022-1032.

13 Feghali-Bostwick CA, Tsai CG, Valentine VG, et al. Cellular and humoral autoreactivity in idiopathic pulmonary fibrosis. J Immunol 2007; 179: 2592-2599.

14 Donahoe M, Valentine VG, Chien N, et al. Autoantibody-targeted treatments for acute exacerbations of idiopathic pulmonary fibrosis. PLoS One 2015; 10: e0127771.

15 Zhou Y, Huang X, Hecker L, et al. Inhibition of mechanosensitive signaling in myofibroblasts ameliorates experimental pulmonary fibrosis. J Clin Invest 2013; 123: 1096-1108.

16 Parker MW, Rossi D, Peterson M, et al. Fibrotic extracellular matrix activates a profibrotic positive feedback loop. J Clin Invest 2014; 124: 1622-1635.

17 Hecker L, Logsdon NJ, Kurundkar D, et al. Reversal of persistent fibrosis in aging by targeting Nox4-Nrf2 redox imbalance. Sci Transl Mede 2014; 6: 231 ra47.

18 Chanda D, Kurundkar A, Rangarajan S, et al. Developmental reprogramming in mesenchymal stromal cells of human subjects with idiopathic pulmonary fibrosis. Sci Rep 2016; 6: 37445.

19 Travis WD, Costabel U, Hansell DM, et al. An official American Thoracic Society/European Respiratory Society statement: update of the international multidisciplinary classification of the idiopathic interstitial pneumonias. Am J Respir Crit Care Med 2013; 188: 733-748.

20 Fingerlin TE, Murphy E, Zhang W, et al. Genome-wide association study identifies multiple susceptibility loci for pulmonary fibrosis. Nat Genet 2013; 45: 613-620.

21 Oldham JM, Ma SF, Martinez FJ, et al. TOLLIP, MUC5B, and the response to N-Acetylcysteine among individuals with idiopathic pulmonary fibrosis. Am J Respir Crit Care Med 2015; 192: 1475-1482.

22 Richards TJ, Kaminski N, Baribaud F, et al. Peripheral blood proteins predict mortality in idiopathic pulmonary fibrosis. Am J Respir Crit Care Med 2012; 185: 67-76.

23 DePianto DJ, Chandriani S, Abbas AR, et al. Heterogeneous gene expression signatures correspond to distinct lung pathologies and biomarkers of disease severity in idiopathic pulmonary fibrosis. Thorax 2015; 70: 48-56. 

increased in plasma of human subjects with interstitial lung disease. Am J Respir Crit Care Med 2016; 193: 861-868.

25 Surolia R, Li FJ, Wang Z, et al. 3D pulmospheres serve as a personalized and predictive multicellular model for assessment of antifibrotic drugs. JCI Insight 2017; 2: e91377.

26 White ES, Xia M, Murray S, et al. Plasma surfactant protein-D, matrix metalloproteinase-7, and osteopontin index distinguishes idiopathic pulmonary fibrosis from other idiopathic interstitial pneumonias. Am J Respir Crit Care Med 2016; 194: 1242-1251.

27 Mathai SK, Newton CA, Schwartz DA, et al. Pulmonary fibrosis in the era of stratified medicine. Thorax 2016; 71: 1154-1160.

28 Spagnolo P, Cottin V. Genetics of idiopathic pulmonary fibrosis: from mechanistic pathways to personalised medicine. J Med Genet 2017; 54: 93-99.

29 Spagnolo P, Tzouvelekis A, Maher TM. Personalized medicine in idiopathic pulmonary fibrosis: facts and promises. Curr Opin Pulm Med 2015; 21: 470-478.

30 Sato S, Kolb MRJ. Personalised medicine for IPF: getting closer, but not there yet. Lancet Respir Med 2017; 5: 836-837.

31 Ramsey BW, Davies J, McElvaney NG, et al. A CFTR potentiator in patients with cystic fibrosis and the G551D mutation. N Engl J Med 2011; 365: 1663-1672.

32 Bel EH, Wenzel SE, Thompson PJ, et al. Oral glucocorticoid-sparing effect of mepolizumab in eosinophilic asthma. N Engl J Med 2014; 371: 1189-1197.

33 Nair P, Wenzel S, Rabe KF, et al. Oral glucocorticoid-sparing effect of benralizumab in severe asthma. $N$ Engl J Med 2017; 376: 2448-2458.

34 Corren J, Lemanske RF, Hanania NA, et al. Lebrikizumab treatment in adults with asthma. N Engl J Med 2011; 365: 1088-1098.

35 Hanania NA, Korenblat P, Chapman KR, et al. Efficacy and safety of lebrikizumab in patients with uncontrolled asthma (LAVOLTA I and LAVOLTA II): replicate, phase 3, randomised, double-blind, placebo-controlled trials. Lancet Respir Med 2016; 4: 781-796.

36 Rosas IO, Richards TJ, Konishi K, et al. MMP1 and MMP7 as potential peripheral blood biomarkers in idiopathic pulmonary fibrosis. PLoS Med 2008; 5: e93.

37 Kahloon RA, Xue J, Bhargava A, et al. Patients with idiopathic pulmonary fibrosis with antibodies to heat shock protein 70 have poor prognoses. Am J Respir Crit Care Med 2013; 187: 768-775.

38 King TE Jr, Bradford WZ, Castro-Bernardini S, et al. A phase 3 trial of pirfenidone in patients with idiopathic pulmonary fibrosis. N Engl J Med 2014; 370: 2083-2092.

39 Raghu G, Wells AU, Nicholson AG, et al. Effect of nintedanib in subgroups of idiopathic pulmonary fibrosis by diagnostic criteria. Am J Respir Crit Care Med 2017; 195: 78-85.

40 Wells AU. Approach to diagnosis of diffuse lung disease. In: Albert RK, Spiro SG, Jett JR, eds. Clinical Respiratory Medicine. 2nd Edn. Mosby, Elsevier Science, 2004.

41 Tashkin DP, Elashoff R, Clements PJ, et al. Cyclophosphamide versus placebo in scleroderma lung disease. N Engl J Med 2006; 354: 2655-2666.

42 Tashkin DP, Roth MD, Clements PJ, et al. Mycophenolate mofetil versus oral cyclophosphamide in scleroderma-related interstitial lung disease (SLS II): a randomised controlled, double-blind, parallel group trial. Lancet Respir Med 2016; 4: 708-719.

43 Fischer A, Brown KK, Du Bois RM, et al. Mycophenolate mofetil improves lung function in connective tissue disease-associated interstitial lung disease. J Rheumatol 2013; 40: 640-646.

44 Morisset J, Johannson KA, Vittinghoff E, et al. Use of mycophenolate mofetil or azathioprine for the management of chronic hypersensitivity pneumonitis. Chest 2017; 151: 619-625.

45 Adegunsoye A, Oldham JM, Fernández Pérez ER, et al. Outcomes of immunosuppressive therapy in chronic hypersensitivity pneumonitis. ERJ Open Res 2017; 3: 00016-2017.

46 Gaxiola M, Buendía-Roldán I, Mejía M, et al. Morphologic diversity of chronic pigeon breeder's disease: clinical features and survival. Respir Med 2011; 105: 608-614

47 Wang P, Jones KD, Urisman A, et al. Pathologic findings and prognosis in a large prospective cohort of chronic hypersensitivity pneumonitis. Chest 2017; 152: 502-509.

48 Pérez-Padilla R, Salas J, Chapela R, et al. Mortality in Mexican patients with chronic pigeon breeder's lung compared with those with usual interstitial pneumonia. Am Rev Respir Dis 1993; 148: 49-53.

49 Ohtani Y, Saiki S, Kitaichi M, et al. Chronic bird fancier's lung: histopathological and clinical correlation. An application of the 2002 ATS/ERS consensus classification of the idiopathic interstitial pneumonias. Thorax 2005; 60: 665-671.

50 Churg A, Sin DD, Everett D, et al. Pathologic patterns and survival in chronic hypersensitivity pneumonitis. Am J Surg Pathol 2009; 33: 1765-1770.

51 Walsh SL, Sverzellati N, Devaraj A, et al. Chronic hypersensitivity pneumonitis: high resolution computed tomography patterns and pulmonary function indices as prognostic determinants. Eur Radiol 2012; 22: $1672-1679$.

52 Park JH, Kim DS, Park IN, et al. Prognosis of fibrotic interstitial pneumonia: idiopathic versus collagen vascular disease-related subtypes. Am J Respir Crit Care Med 2007; 175: 705-711.

53 Tsuchiya Y, Takayanagi N, Sugiura H, et al. Lung diseases directly associated with rheumatoid arthritis and their relationship to outcome. Eur Respir J 2011; 37: 1411-1417.

54 Moua T, Zamora Martinez AC, Baqir M, et al. Predictors of diagnosis and survival in idiopathic pulmonary fibrosis and connective tissue disease-related usual interstitial pneumonia. Respir Res 2014; 15: 154.

55 Nakamura Y, Suda T, Kaida Y, et al. Rheumatoid lung disease: prognostic analysis of 54 biopsy-proven cases. Respir Med 2012; 106: 1164-1169.

56 Song JW, Lee HK, Lee CK, et al. Clinical course and outcome of rheumatoid arthritis-related usual interstitial pneumonia. Sarcoidosis Vasc Diffuse Lung Dis 2013; 30: 103-112.

57 Kim EJ, Elicker BM, Maldonado F, et al. Usual interstitial pneumonia in rheumatoid arthritis-associated interstitial lung disease. Eur Respir J 2010; 35: 1322-1328. 

prognostic factors and physiological and radiological characteristics-a large multicentre UK study. Rheumatology (Oxford) 2014; 53: 1676-1682.

59 Solomon JJ, Chung JH, Cosgrove GP, et al. Predictors of mortality in rheumatoid arthritis-associated interstitial lung disease. Eur Respir J 2016; 47: 588-596.

60 Yunt ZX, Chung JH, Hobbs S, et al. High resolution computed tomography pattern of usual interstitial pneumonia in rheumatoid arthritis-associated interstitial lung disease: Relationship to survival. Respir Med 2017; 126: $100-104$.

61 Nurmi HM, Kettunen HP, Suoranta SK, et al. Several high-resolution computed tomography findings associate with survival and clinical features in rheumatoid arthritis-associated interstitial lung disease. Respir Med 2018; 134: 24-30.

62 Fischer A, Antoniou KM, Brown KK, et al. An official European Respiratory Society/American Thoracic Society research statement: interstitial pneumonia with autoimmune features. Eur Respir J 2015; 46: 976-987.

63 Oldham JM, Adegunsoye A, Valenzi E, et al. Characterisation of patients with interstitial pneumonia with autoimmune features. Eur Respir J 2016; 47: 1767-1775.

64 Assayag D, Elicker BM, Urbania TH, et al. Rheumatoid arthritis-associated interstitial lung disease: radiologic identification of usual interstitial pneumonia pattern. Radiology 2014; 270: 583-588.

65 Jacob J, Bartholmai BJ, Rajagopalan S, et al. Unclassifiable-interstitial lung disease: Outcome prediction using CT and functional indices. Respir Med 2017; 130: 43-51.

66 Ryerson CJ, Urbania TH, Richeldi L, et al. Prevalence and prognosis of unclassifiable interstitial lung disease. Eur Respir J 2013; 42: 750-757.

67 Thomeer MJ, Vansteenkiste J, Verbeken EK, et al. Interstitial lung diseases: characteristics at diagnosis and mortality risk assessment. Respir Med 2004; 98: 567-573.

68 Latsi PI, du Bois RM, Nicholson AG, et al. Fibrotic idiopathic interstitial pneumonia: the prognostic value of longitudinal functional trends. Am J Respir Crit Care Med 2003; 168: 531-537.

69 Jegal Y, Kim DS, Shim TS, et al. Physiology is a stronger predictor of survival than pathology in fibrotic interstitial pneumonia. Am J Respir Crit Care Med 2005; 171: 639-644.

70 Sleijfer S. Bleomycin-induced pneumonitis. Chest 2001; 120: 617-624.

71 Nardi A, Brillet PY, Letoumelin P, et al. Stage IV sarcoidosis: comparison of survival with the general population and causes of death. Eur Respir J 2011; 38: 1368-1373.

72 Gimenez A, Storrer K, Kuranishi L, et al. Change in FVC and survival in chronic fibrotic hypersensitivity pneumonitis. Thorax 2018; 73: 391-392.

73 Goh NS, Hoyles RK, Denton CP, et al. Short-term pulmonary function trends are predictive of mortality in interstitial lung disease associated with systemic sclerosis. Arthritis Rheumatol 2017; 69: 1670-1678.

74 Kulkarni T, de Andrade J, Zhou Y, et al. Alveolar epithelial disintegrity in pulmonary fibrosis. Am J Physiol Lung Cell Mol Physiol 2016; 311: L185-L191.

75 Cao Z, Lis R, Ginsberg M, et al. Targeting of the pulmonary capillary vascular niche promotes lung alveolar repair and ameliorates fibrosis. Nat Med 2016; 22: 154-162.

76 Juge PA, Borie R, Kannengiesser C, et al. Shared genetic predisposition in rheumatoid arthritis-interstitial lung disease and familial pulmonary fibrosis. Eur Respir J 2017; 49: 1602314.

77 Snetselaar R, van Batenburg AA, van Oosterhout MFM, et al. Short telomere length in IPF lung associates with fibrotic lesions and predicts survival. PLoS One 2017; 12: e0189467.

78 Ley B, Newton CA, Arnould I, et al. The MUC5B promoter polymorphism and telomere length in patients with chronic hypersensitivity pneumonitis: an observational cohort-control study. Lancet Respir Med 2017; 5: 639-647.

79 Kennedy B, Branagan P, Moloney F, et al. Biomarkers to identify ILD and predict lung function decline in scleroderma lung disease or idiopathic pulmonary fibrosis. Sarcoidosis Vasc Diffuse Lung Dis 2015; 32: $228-236$.

80 Luckhardt TR, Thannickal VJ. Systemic sclerosis-associated fibrosis: an accelerated aging phenotype? Curr Opin Rheumatol 2015; 27: 571-576.

81 Thannickal VJ. Mechanistic links between aging and lung fibrosis. Biogerontology 2013; 14: 609-615.

82 Chilosi M, Doglioni C, Murer B, et al. Epithelial stem cell exhaustion in the pathogenesis of idiopathic pulmonary fibrosis. Sarcoidosis Vasc Diffuse Lung Dis 2010; 27: 7-18.

83 Birch J, Barnes PJ, Passos JF. Mitochondria, telomeres and cell senescence: Implications for lung ageing and disease. Pharmacol Ther 2018; 183: 34-49.

84 Stock CJ, Sato H, Fonseca C, et al. Mucin 5B promoter polymorphism is associated with idiopathic pulmonary fibrosis but not with development of lung fibrosis in systemic sclerosis or sarcoidosis. Thorax 2013; 68: 436-441.

85 Peljto AL, Zhang Y, Fingerlin TE, et al. Association between the MUC5B promoter polymorphism and survival in patients with idiopathic pulmonary fibrosis. JAMA 2013; 309: 2232-2239.

86 Noth I, Zhang Y, Ma SF, et al. Genetic variants associated with idiopathic pulmonary fibrosis susceptibility and mortality: a genome-wide association study. Lancet Respir Med 2013; 1: 309-317.

87 Behr J, Neuser P, Prasse A, et al. Exploring efficacy and safety of oral pirfenidone for progressive, non-IPF lung fibrosis (RELIEF) - a randomized, double-blind, placebo-controlled, parallel group, multi-center, phase II trial. BMC Pulm Med 2017; 17: 122.

88 Flaherty KR, Brown KK, Wells AU, et al. Design of the PF-ILD trial: a double-blind, randomised, placebo-controlled phase III trial of nintedanib in patients with progressive fibrosing interstitial lung disease. BMJ Open Respir Res 2017; 4: e000212.

89 Hamman L, Rich AR. Fulminating diffuse interstitial fibrosis of the lungs. Trans Am Clin Climat Assoc 1935; 51: $154-163$.

90 Liebow AA. New concepts and entities in pulmonary disease. In: Liebow AA, Smith DE, eds. The Lung. Baltimore, The Williams and Wilkins Company; 1968; pp. 332-365.

91 Carrington CB, Gaensler EA, Coutu RE, et al. Natural history and treated course of usual and desquamative interstitial pneumonia. N Engl J Med 1978; 298: 801-809.

92 Grinblat J, Mechlis S, Lewitus Z. Organizing pneumonia-like process: an unusual observation in steroid responsive cases with features of chronic interstitial pneumonia. Chest 1981; 80: 259-263. 

382-394.

94 Epler GR, Colby TV, McLoud TC, et al. Bronchiolitis obliterans organizing pneumonia. N Engl J Med 1985; 312: $152-158$.

95 Crystal RG, Bitterman PB, Rennard SI, et al. Interstitial lung diseases of unknown cause: disorders characterized by chronic inflammation of the lower respiratory tract. N Engl J Med 1984; 310: 154-166.

96 Ohori NP, Sciurba FC, Owens GR, et al. Giant-cell interstitial pneumonia and hard-metal pneumoconiosis. a clinicopathologic study of four cases and review of the literature. Am J Surg Pathol 1989; 13: 581-587.

97 Abraham JL, Burnett BR, Hunt A. Development and use of a pneumoconiosis database of human pulmonary inorganic particulate burden in over 400 lungs. Scanning Microsc 1991; 5: 95-104.

98 Katzenstein ALA, Fiorelli RF. Nonspecific interstitial pneumonia/fibrosis. Histological features and clinical significance. Am J Surg Pathol 1994; 18: 136-147.

99 American Thoracic Society. Idiopathic pulmonary fibrosis: diagnosis and treatment. International consensus statement. American Thoracic Society (ATS), and the European Respiratory Society (ERS). Am J Respir Crit Care Med 2000; 161: 646-664.

100 Beasley MB, Franks TJ, Galvin JR, et al. Acute fibrinous and organizing pneumonia: a histological pattern of lung injury and possible variant of diffuse alveolar damage. Arch Pathol Lab Med 2002; 126: 1064-1070.

101 Cottin V, Nunes H, Brillet PY, et al. Combined pulmonary fibrosis and emphysema: a distinct underrecognised entity. Eur Respir J 2005; 26: 586-593.

102 Armanios MY, Chen JJL, Cogan JD, et al. Telomerase mutations in families with idiopathic pulmonary fibrosis. N Engl J Med 2007; 356: 1317-1326.

103 Richeldi L, du Bois RM, Raghu G, et al. Efficacy and safety of nintedanib in idiopathic pulmonary fibrosis. N Engl J Med 2014; 370: 2071-2082.

104 Raghu G, Rochwerg B, Zhang Y, et al. An Official ATS/ERS/JRS/ALAT Clinical Practice Guideline: Treatment of Idiopathic Pulmonary Fibrosis. An Update of the 2011 Clinical Practice Guideline. Am J Respir Crit Care Med 2015; 192: e3-e19.

105 Lynch DA, Sverzellati N, Travis WD, et al. Diagnostic criteria for idiopathic pulmonary fibrosis: a Fleischner Society White Paper. Lancet Respir Med 2018; 6: 138-153. 